PublisherName : BioMed Central

PublisherLocation : London

PublisherlmprintName : BioMed Central

\title{
Assessment of ventriculo-vascular properties in repaired coarctation using cardiac MRI derived aortic, left atrial and left ventricular strain
}

\begin{tabular}{|c|c|}
\hline & Articlel nfo \\
\hline Articlel D & : 4748 \\
\hline ArticleDOI & : 10.1186/1532-429X-17-S1-P207 \\
\hline ArticleCitationID & : P207 \\
\hline ArticleSequenceNumber & : 707 \\
\hline ArticleCategory & : Poster presentation \\
\hline ArticleFirstPage & $: 1$ \\
\hline ArticleLastPage & $: 1$ \\
\hline ArticleHistory & : RegistrationDate : 2015-2-3 \\
\hline & OnlineDate $\quad:$ 2015-2-3 \\
\hline ArticleCopyright & $\begin{array}{l}\text { : Shang et al; licensee BioMed Central Ltd.2015 } \\
\text { This article is published under license to BioMed Central Ltd. This is an Open } \\
\text { Access article distributed under the terms of the Creative Commons Attribution } \\
\text { License (http://creativecommons.org/licenses/by/4.0), which permits } \\
\text { unrestricted use, distribution, and reproduction in any medium, provided the } \\
\text { original work is properly cited. The Creative Commons Public Domain } \\
\text { Dedication waiver (http://creativecommons.org/publicdomain/zero/1.0/) } \\
\text { applies to the data made available in this article, unless otherwise stated. }\end{array}$ \\
\hline ArticleGrants & : \\
\hline ArticleContext & : 129681717S1S1 \\
\hline
\end{tabular}


Quanliang Shang,Aff1

Shivani Patel, Aff1

Phalla Ou, Aff2

David Danford, Aff1

Andreas Schuster, Aff3

Philipp B Beerbaum, Aff4

Samir Sarikouch, Aff4

Shelby Kutty, Aff1

Corresponding Affiliation: Aff1

Aff1 University of Nebraska Medical Center, Chiildren's Hospital and Medical

Center, Omaha, NE, USA

Aff2 University Paris Diderot, Hopital Bichat, APHP, Paris, France

Aff3 University Medical Center, Göttingen, Göttingen, Germany

Aff4 Hanover Medical University, Hannover, Hannover, Germany

\section{Background}

Impaired arterial strain is associated with increased stiffness in the great arteries. In patients with repaired coarctation of the aorta (COA), we sought to assess ventriculo-vascular characteristics using cardiac magnetic resonance (CMR) derived aortic area strain (AS) in conjunction with left atrial (LA) and left ventricular (LV) longitudinal and circumferential strain (LS and CS).

\section{Methods}

Seventy five subjects, including 50 patients with repaired CoA divided into hypertensive $(n=25)$, and normotensive groups ( $n=25$ ), and 25 healthy subjects (controls) were studied. Hypertension was defined per National High Blood Pressure Education Program Working Group guidelines as systolic blood pressure (SBP) $\geq 95$ th percentile. For aorta acquisitions, through-plane, free-breathing, phase-contrast CMR protocol was used and AS measured at 3 levels: ascending aorta, proximal descending aorta and descending aorta at diaphragm. Maximal and minimal luminal areas $\left(\mathrm{mm}^{2}\right)$ were measured, and AS (\%) was calculated as 100 X (Area max - Area min)/ Area min). LA and LV LS were measured from horizontal long axis steady state free precession cine (4-chamber image) and LVCS from mid-ventricular short axis cine using CMRfeature tracking (6-segment model, 2D-CPA, TomTec). LA and LV end-diastolic volumes (EDV), ejection fraction (EF) and LV mass were also measured (Medis) in all subjects. Measurements were compared between the 3 groups. 


\section{Results}

The mean age of CoA patients were $19.7 \pm 6.7$ years (range 8.5-43 years) and controls were $23 \pm 15$ years (range 7.1-51 years). All strains (LA, LV, ascending and descending aortic) were significantly lower in CoA subgroups compared to controls (Figure 1) except the AS at diaphragm which was not different between CoA subgroups and controls. Comparisons between the hypertensive and normotensive CoA subgroups showed no statistically significant differences in indexed LV mass, LA and LV volumetric and strain indices, however the ascending AS was significantly lower in the hypertensive subgroup $(p=0.02)$. Ascending AS was correlated with LV mass $(r=-0.4, p=0.005), \operatorname{LVEF}(r=-0.4, p=0.004), \operatorname{SBP}(r=-0.5$, $p=0.000)$ and $\operatorname{LVL}(r=0.5, p=0.001)$.

Figure 1

\begin{tabular}{|c|c|c|c|c|}
\hline & $\begin{array}{c}\text { Normotensive } \\
\text { CoA }\end{array}$ & $\begin{array}{c}\text { Hypertensive } \\
\text { CoA }\end{array}$ & Controls & $p$ \\
\hline & $\mathrm{n}=25$ & $\mathrm{n}=25$ & $\mathrm{n}=25$ & \\
\hline Gender (M:F) & $14: 11$ & $14: 11$ & $14: 11$ & 0.31 \\
\hline Age(years) & $19 \pm 7.4$ & $21 \pm 6$ & $23 \pm 15$ & $<0.001$ \\
\hline SBP(mmHg) & $118.8 \pm 7$ & $137.4 \pm 11$ & $117.5 \pm 6$ & 0.10 \\
\hline DBP(mmHg) & $65 \pm 8$ & $69 \pm 10$ & $64 \pm 6$ & 0.03 \\
\hline $\begin{array}{c}\text { Indexed LV mass } \\
\left(\mathrm{g} / \mathrm{m}^{2}\right)\end{array}$ & $58 \pm 18$ & $66 \pm 14$ & $54 \pm 10$ & 0.002 \\
\hline LV mass(mg) & $95 \pm 32$ & $122 \pm 35$ & $89 \pm 33$ & 0.35 \\
\hline $\begin{array}{c}\text { Indexed LVEDV } \\
\text { (ml/m })\end{array}$ & $79 \pm 16$ & $83 \pm 12$ & $78 \pm 12$ & 0.004 \\
\hline LVEF(\%) & $62 \pm 7$ & $65 \pm 6$ & $59 \pm 4$ & 0.007 \\
\hline LALS(\%) & $20 \pm 9$ & $21 \pm 7$ & $27 \pm 7$ & 0.003 \\
\hline LVLS(\%) & $16.6 \pm 3.7$ & $15.9 \pm 3.7$ & $19.7 \pm 4.8$ & $<0.06$ \\
\hline LVCS(\%) & $23 \pm 4.2$ & $24 \pm 5$ & $27 \pm 4.1$ & $<0.001$ \\
\hline Ascending AS(\%) & $30 \pm 8.2$ & $24 \pm 9.4$ & $46 \pm 11$ & 0.55 \\
\hline $\begin{array}{c}\text { Proximal descending } \\
\text { AS (\%) }\end{array}$ & $32 \pm 11.3$ & $28 \pm 15.7$ & $42 \pm 6$ & $43 \pm 8$ \\
\hline $\begin{array}{c}\text { Diaphragm } \\
\text { descending AS (\%) }\end{array}$ & $42 \pm 13$ & $39 \pm 16$ & & $<$ \\
\hline
\end{tabular}

\section{Conclusions}

Ascending AS in CoA correlated with LV mass, EF and LVLS. In hypertensive CoA subjects, ascending AS was significantly reduced compared to normotensive CoA and healthy controls of similar age and body surface area. These observations may be indicative of differences in vascular remodeling influenced by ongoing hypertension.

\section{Funding}

Childrens Hospital and Medical Center Foundation, Omaha, USA. 